\title{
Comunicação
}

\section{Estudo da densidade óssea na esclerodermia sistêmica}

\author{
H.C. DA Silva, V.L. Szej nfeld, L.S.S. Assis, E.I. Sato \\ Disciplina de Reumatologia da Universidade Federal de São Paulo - Escola Paulista de M edicina, São Paulo, SP.
}

\begin{abstract}
RESUMO - Овj ETIvo. A osteopenia em pacientes com esclerodermia sistêmica foi descrita, radiologicamente, em mãos e, por densidade óssea, no terço proximal e distal do rádio. A redução da massa óssea, nesses pacientes, tem sido atribuída à isquemia, imobilização e à menopausa precoce. 0 objetivo deste estudo é analisar a densidade óssea na coluna, região proximal do fêmur e corpo todo de pacientes com esclerodermia sistêmica.

Pacientes e Método. Foram examinadas 25 pacientes caucasóides, sem outras condições que pudessem afetar o metabolismo ósseo. A média de idade das pacientes foi de $48 \pm 12$ anos, e o tempo de doença, de $7 \pm 7$ anos; 13 estavam na pós-menopausa há $8 \pm 8$ anos. A medida de massa óssea foi realizada na coluna, região proximal do fêmur e corpo todo, utilizando-se densitômetro de dupla emissão com fonte de raios X (Lunar - modelo DPX).
\end{abstract}

\section{INTRODUÇÃO}

O envolvimento ósseo, em pacientes com esclerodermia sistêmica, caracteriza-se, geralmente, por reabsorção óssea, predominantemente das extremi dades das falanges distais dos dedos das mãos e, mais raramente, dos pés e outras partes do esqueleto1-3. Também, já foi descrito, em pacientes com esclerodermia, principalmente em mãos, osteopenia localizada periarticular, que, nos estágios mais tardi os da doença, pode estender-se por todo o membro comprometido ${ }^{4-6}$.

A observação inicial, associando osteopenia generalizada à esclerodermia, foi feita por Serup et al. ${ }^{5}$, em 1983. Esses autores, utilizando densitometria de emissão única, observaram redução do conteúdo mineral ósseo do terço distal do rádio em dez (29\%) dos 37 pacientes (30 mulheres e sete homens) estudados. Verificaram, também, que a osteopenia foi mais freqüente nos pacientes que tinham calcinose, e que os parâmetros bioquímicos (cálcio, fósforo, fosfatase alcalina, hormônio paratiroidiano [PTH] séricos e cálcio urinário) não foram diferentes dos obtidos no grupo-controle.

La M ontagna et al. ${ }^{6}$, em 1991, ao estudarem, com densitômetro de dupla emissão, a densi dadeóssea da região distal e proximal do antebraço de 90 mulheres
Resultados. Não houve diferença estatisticamente significante na densidade óssea das regiões avaliadas nas pacientes com esclerodermia sistêmica e as mulheres-controle pareadas para a idade, peso, altura e anos de menopausa. A densidade óssea das pacientes com forma limitada não foi diferente daquelas com a forma difusa. Pacientes com calcinose apresentaram menor densidade óssea na região proximal do fêmur que aquelas sem calcinose.

ConClusões. Os autores concluíram que pacientes com esclerodermia sistêmica não apresentam perda de massa óssea. Portanto, a esclerodermia não é um fator de risco para o desenvolvimento de osteoporose generalizada.

UNITERMOS. Densidade óssea. Osteoporose. Esclerodermia sistêmica.

com esclerodermia, observaram que todas as pacientes apresentavam di mi nui ção significativa da densidade óssea, quando comparadas ao grupo-controle. Nenhuma das pacientes estudadas apresentava comprometimento renal e/ou síndrome de má-absorção e não estavam em uso de corticosteróides, porém, foram incluídas mulheres na pré e pós-menopausa. La Montagna et al ${ }^{6}$, da mesma forma que Serup et al. ${ }^{5}$, também não encontraram quaisquer alterações nos parâmetros bioquímicos referentes ao metabolismo de cál ci o e fósforo. Não observaram correl ação significante da densidade óssea com o tempo de evolução, com a extensão do comprometimento dérmico, com a presença de calcinose, com a ocorrência de artrite e alterações viscerais. Entretanto, La Montagna et al. ${ }^{6}$ verificaram que o grupo de pacientes estudadas apresentou mai or inci dência de menopausa precoce, e que aquelas que estavam na prémenopausa não apresentavam redução da massa óssea, quando comparadas ao grupo-controle. Os autores concluíram que a menopausa foi mais importante na diminuição da densidade óssea do que a esclerodermia propriamente dita.

E mbora, até o momento, apenas esses autores ${ }^{5,6}$ tenham estudado a associação entre escl erodermia e osteoporose, alguns aspectos clínicos, fisiopatológicos e radiológicos, característicos da esclerodermia 
sistêmica, sugerem a possibilidade de que a osteoporose possa estar presente nesses pacientes.

A osteopenia, em pacientes com esclerodermia sistêmica, poderia ser primária, isto é, relacionada aos processos fisiopatológicos inerentes à doença, ou secundária à artropatia inflamatória, à imobilização e desuso decorrentes do endurecimento da pele ou ao comprometimento muscular freqüentemente encontrado nesses pacientes. Além disso, a síndrome de má-absorção, encontrada em 10\% dos pacientes, e o comprometimento renal, que pode evoluir para insuficiência renal crônica ${ }^{7}$, também poderiam levar à osteopenia.

Tendo em vista que os dados na literatura sugerindo a presença de osteopenia em pacientes com esclerodermia são, ainda, controvertidos e foram real izados apenas no terço proximal e distal do rádio, o objetivo do presente estudo é avaliar a densidade óssea da coluna, região proximal do fêmur e do corpo todo de pacientes com esclerodermia, pois essas regiões do esqueleto, freqüentemente, apresentamse alteradas em pacientes com osteoporose.

\section{PACIENTES E MÉTODO}

Foram estudados pacientes com esclerodermia acompanhados no Ambulatório da Disciplina de Reumatologia, do Departamento de Medicina, da Escola Paulista de Medicina, durante os anos de 1990 a 1992.

Todas eram do sexo feminino, caucasóides e preenchiam os critérios diagnósticos propostos pelo ACR (American College of Rheumatology) em $1980^{8}$. Foram excluídas do estudo aquelas que apresentassem doença hepática, doença gastrointestinal de qualquer natureza, doença renal, comprometimento pulmonar, clinicamente detectável, diabetes melito, alcool ismo, doenças da tirói de e/ou paratiróide, dieta vegetariana, corticoterapia nos últimos 12 meses e/ ou tratamento com drogas que, sabidamente, alterem o metabolismo ósseo, tais como anticonvulsivantes, heparina, metaból itos da vitamina $D$, reposi ção hormonal, supl emento de cál cio, antiácidos com alumínio e anticoncepcionais nos últimos 12 meses que antecederam o estudo.

Das 32 pacientes examinadas, 25 preencheram os critérios acima propostos e entraram para o presente estudo.

Todas as pacientes foram avaliadas clinica e fisicamente pelo mesmo observador (HCS) e responderam a um protocolo previamente estabelecido, em que foram anotados os principais aspectos sobre hábitos alimentares, atividade física, fumo e ingestão de álcool. Foram, também, assinalados os antecedentes ginecológicos e obstétricos, tais como: primeir ra menstruação, regulari dade dos cicl os menstruais, última menstruação, número de filhos, abortamentos e tempo de amamentação.

O sangue e a urina de 24 horas foram obtidos dois a três dias antes da realização da medida de densidade óssea. Foram dosados cálcio, fósforo, fosfatase alcalina e creatinina séricas, utilizando-se métodos padronizados. O cálcio na urina de 24 horas foi coletado em frascos contendo $20 \mathrm{~mL}$ de ácido cl orídrico a $50 \%$ e medido por absorção atômica.

As pacientes estudadas foram submetidas a radiografias simples de mãos e punhos em projeção ântero-posterior, quando foram medidos o índice de Nordin ${ }^{9}$ e avaliadas as princi pais alterações radiológicas decorrentes da esclerodermia sistêmica.

As densidades ósseas da coluna lombar, região proximal do fêmur (colo, triângulo de Ward e trocanter) e do corpo inteiro foram medi das utilizandose densitômetro de dupla emissão com fonte de raios $X$ (Lunar Radiation Corporation, Madison, WI, modelo DPX). Em nosso laboratório, o coeficiente de variação interexames é de 1,8\% para a col una, 2,9\% para o col o do fêmur, 3,5\% para o triângulo de Ward e 3,1\% para o trocanter ${ }^{10}$.

Para controle da normalidade da col una e região proximal do fêmur, mediu-se a densidade óssea, nessas regiões, de 90 mulheres saudáveis, e o controle de normal idade da densidade óssea do corpo inteiro foi real izado em 32 mulheres saudáveis. Todas as mulheres do grupo-controle eram brasileiras, caucasóides, com idade, peso e altura semelhantes às das pacientes estudadas.

O teste $t$ deStudent, para amostras não-pareadas, foi utilizado para a comparação entre as densidades ósseas dos diversos locais examinados das pacientes e das mulheres-controles saudáveis agrupadas pela idade e anos de menopausa. O mesmo teste foi empregado para a comparação das densidades ósseas dos diferentes locais estudados entre as pacientes com forma limitada e difusa, pacientes com e sem alterações radiológicas relacionadas à esclerodermia sistêmica e para a comparação entre aquelas com e sem osteoporose cortical em mãos.

Para correl acionar a idade, os anos de menopausa e o tempo de evolução da doença com as densidades ósseas das diferentes regiões estudadas, foi utilizado o teste de correlação de Pearson.

O nível de significância escol hido foi de $p<0,05$.

\section{RESULTADOS}

A média de idade das pacientes foi de $48 \pm 12$ anos(22 a 72), o peso médi o, de $66 \pm 10 \mathrm{~kg}$ (48 a 86), a altura, de 1,57 $\pm 0,06 \mathrm{~m}$ ( 1,46 a 1,74), o índice de massa corpórea, calculado pela fórmula de Quetelet 
Tabela 1 - Densidade óssea $\left(\mathrm{g} / \mathrm{cm}^{2}\right)$ da coluna, região proximal do fêmur (colo, triângulo de Ward, trocanter) e corpo inteiro das pacientes e do grupo-controle, quando agrupadas pela idade $(X \pm D P)$

\begin{tabular}{|c|c|c|c|c|c|c|}
\hline & \multicolumn{2}{|c|}{$<40$ anos } & \multicolumn{2}{|c|}{ entre 40 e 50 anos } & \multicolumn{2}{|c|}{$>50$ anos } \\
\hline & $\begin{array}{c}P a c \\
(n=6)\end{array}$ & $\begin{array}{c}\text { Ctl } \\
(n=24)\end{array}$ & $\begin{array}{c}\text { Pac } \\
(n=6)\end{array}$ & $\underset{(n=35)}{C}$ & $\begin{array}{c}\text { Pac } \\
(n=13)\end{array}$ & $\begin{array}{l}\text { Ctl } \\
(n=31)\end{array}$ \\
\hline Coluna & $1,24 \pm, 29$ & $1,14 \pm, 08$ & $1,03 \pm, 13$ & $1,13 \pm, 17$ & $1,03 \pm, 18$ & $1,00 \pm, 16$ \\
\hline Colo & $1,04 \pm, 08$ & $0,91 \pm, 09$ & $0,86 \pm, 18$ & $0,87 \pm, 11$ & $0,80 \pm, 14$ & $0,79 \pm, 10$ \\
\hline Ward & $1,05 \pm, 45$ & $0,85 \pm, 10$ & $0,75 \pm, 25$ & $0,74 \pm, 13$ & $0,65 \pm, 16$ & $0,65 \pm, 11$ \\
\hline Trocanter & $0,81 \pm, 23$ & $0,75 \pm, 08$ & $0,74 \pm, 13$ & $0,71 \pm, 11$ & $0,66 \pm, 09$ & $0,67 \pm, 10$ \\
\hline \multirow[t]{2}{*}{ Corpo inteiro } & $1,07 \pm, 18$ & $1,04 \pm, 13$ & $1,12 \pm, 13$ & $1,12 \pm, 09$ & $1,03 \pm, 09$ & $1,07 \pm, 10$ \\
\hline & $(n=6)$ & $(n=8)$ & $(\mathrm{n}=6)$ & $(n=12)$ & $(n=11)$ & $(n=12)$ \\
\hline
\end{tabular}

Pac. $=$ pacientes $;$ Ctl. $=$ controles

(peso/altura ${ }^{2}$ ), de $27 \pm 4 \mathrm{~kg} / \mathrm{m}^{2}$ (21 a 34) e o tempo médi o de evol ução da doença foi de $7 \pm 7$ anos ( 1 a 24). Treze pacientes estavam na menopausa há $8 \pm 8$ anos (1 a 30). Duas pacientes tinham história de menopausa precoce.

A forma clínica limitada predominou, estando presente em 19 das 25 pacientes estudadas. Quatro apresentavam esclerodermia difusa e duas, a forma intermediária de Giordano ${ }^{11}$.

Os comprometimentos clínicos mais freqüentes foram esclerodactilia, presente em $94 \%$ das pacientes, fenômeno de Raynaud, presente em $88 \%$, e dismotilidade esofagiana em $84 \%$.

As características clínicas e demográficas das pacientes e do grupo-controle, quando divididas pela idade, foram semelhantes. As médias das densidades ósseas na coluna, região proximal do fêmur e corpo inteiro das pacientes não foram estatisticamente diferentes do grupo-controle para nenhuma faixa etária (tabela 1).

As características clínicas e demográficas das pacientes e do grupo-controle, quando agrupadas de acordo com os anos de menopausa, foram semel hantes para todos os grupos. As médias das densidades ósseas na coluna, região proximal do fêmur e corpo inteiro das pacientes foram semelhantes às encontradas no grupo-controle, em todos os grupos, de acordo com os anos de menopausa.

Houve correlação negativa estatisticamente significante das densidades ósseas do colo do fêmur, triângulo de Ward e trocanter com a idade, indicando que há diminuição da densidade óssea com o aumento da idade. No entanto, não houve qualquer correlação das densidades ósseas com os anos de menopausa. Houve correlação negativa estatisticamente significante do tempo de doença apenas com a densidade óssea do colo do fêmur (tabela 2).

Ao se comparar as densi dades ósseas das pacientes com a forma limitada com aquelas com forma difusa, também não se observou diferença estatisticamente significante para nenhuma região estudada.

\begin{tabular}{|c|c|c|c|c|c|}
\hline & $\begin{array}{l}\text { D.O. } \\
\text { coluna }\end{array}$ & $\begin{array}{l}\text { D.O. } \\
\text { colo }\end{array}$ & $\begin{array}{l}\text { D.O. } \\
\text { Ward }\end{array}$ & $\begin{array}{l}\text { D.O. } \\
\text { trocanter }\end{array}$ & $\begin{array}{c}\text { D.O. } \\
\text { corpo todo }\end{array}$ \\
\hline Idade anos & $0,402^{*}$ & $0,535^{*}$ & $0,575^{\star}$ & $0,489^{*}$ & 0,179 \\
\hline Anos menopausa & 0,308 & 0,260 & 0,328 & 0,232 & 0,251 \\
\hline Anos de doença & 0,346 & $0,439^{*}$ & 0,374 & 0,379 & 0,291 \\
\hline
\end{tabular}

Tabela 3 - Relação entre os achados radiológicos relacionados à esclerodermia e às densidades ósseas $\left(\mathrm{g} / \mathrm{cm}^{2}\right)$ encontradas na coluna, região proximal do fêmur (colo, triângulo de Ward, trocanter) e do corpo inteiro das 25 pacientes estudadas $(X \pm D P)$

\begin{tabular}{|c|c|c|c|}
\hline & \multicolumn{2}{|c|}{$\begin{array}{l}\text { Achados radiológicos } \\
\text { Reabsorção óssea de falanges distais }\end{array}$} & \multirow[b]{2}{*}{$\mathbf{p}$} \\
\hline & $\begin{array}{c}\text { Presente } \\
(n=12)\end{array}$ & $\begin{array}{c}\text { Ausente } \\
(n=13)\end{array}$ & \\
\hline Coluna & $1,08 \pm 0,23$ & $1,09 \pm 0,20$ & ns \\
\hline Colo & $0,89 \pm 0,21$ & $0,86 \pm 0,18$ & ns \\
\hline Ward & $0,82 \pm 0,36$ & $0,73 \pm 0,25$ & ns \\
\hline Trocanter & $0,75 \pm 0,15$ & $0,70 \pm 0,16$ & ns \\
\hline \multirow[t]{3}{*}{ Corpo inteiro } & $1,05 \pm 0,11$ & $1,04 \pm 0,15$ & ns \\
\hline & \multicolumn{2}{|c|}{ Calcificações extra-ósseas } & \\
\hline & $\begin{array}{c}\text { Presente } \\
(n=5)\end{array}$ & $\begin{array}{c}\text { Ausente } \\
(n=20)\end{array}$ & p \\
\hline Coluna & $0,97 \pm 0,13$ & $1,11 \pm 0,23$ & ns \\
\hline Colo & $0,77 \pm 0,12$ & $0,90 \pm 0,20$ & * \\
\hline Ward & $0,60 \pm 0,16$ & $0,82 \pm 0,32$ & * \\
\hline Trocanter & $0,65 \pm 0,10$ & $0,74 \pm 0,16$ & * \\
\hline Corpo inteiro & $0,99 \pm 0,05$ & $1,05 \pm 0,15$ & ns \\
\hline
\end{tabular}

O cálcio, fósforo, creatinina e fosfatase alcalina séricas de todas as pacientes estavam dentro dos limites da normali dade. Dezesseis real izarama dosagem de cál cio na urina de 24 horas, que também se apresentou dentro dos limites da normalidade.

Quanto às alterações radiológicas relacionadas à esclerodermia, 50\% das pacientes tinham reabsorção 
das falanges distais, $21 \%$ calcificações de partes moles e $2 \%$ erosões articulares. Em relação às alterações radiológicas relacionadas à osteoporose, apenas seis (25\%) apresentaram índice de N ordin menor que 0,72 , ou seja, osteopenia cortical.

Ao se comparar as alterações radiológicas relacionadas à esclerodermia com as densidades ósseas medidas nos diferentes locais, observou-se que as pacientes com calcificação de partes moles apresentavam menor massa óssea no colo do fêmur, triângulo de Ward e trocanter, quando comparadas àquel as sem calcinose, não tendo sido verificada qualquer relação da massa óssea com as outras manifestações radiológicas pesquisadas (tabela 3 ).

\section{DISCUSSÃO}

Os resultados deste estudo indicam que a massa óssea da coluna, região proximal do fêmur e corpo inteiro de pacientes com esclerodermia sistêmica não difere da massa óssea de mulheres-controle saudáveis.

Como assinalado anteriormente, Serup et al. ${ }^{5}$ observaram redução da massa óssea em $27 \%$ das pacientes com esclerodermia, e La Montagna et al. ${ }^{6}$ descreveram que pacientes com essa doença apresentaram menor média da densidade óssea, quando comparadas aos controles normais.

A diferença entre nossos resultados pode ter ocorrido porque os autores mencionados mediram o conteúdo mineral ósseo do terço proximal e/ou distal do rádio, regiões ricas em osso cortical e pobre em trabecular ${ }^{12}$. Além disso, as proporções entre osso cortical e trabecular sofrem variações significativas, dependendo do modo de aplicação das técnicas utilizadas, o que pode comprometer a precisão e a reprodutibilidade das medidas obtidas no rádio ${ }^{13-15}$.

Outro aspecto a ser assinalado é o fato de diferentes autore ${ }^{14-16}$ terem observado baixa correlação entre as densidades ósseas medidas no terço proximal e/ou distal do rádio com a coluna e a região proximal do fêmur. Da mesma forma, Aloia et al. ${ }^{16}$, em 1990, ao estudarem mulheres saudáveis, também não encontraram correlação entre a densidade óssea do terço distal do rádio e o conteúdo do cál cio corporal total.

A diminuição da massa óssea com o aumento da idade, observada em nossa casuística, está de acordo com o descrito por outros autores. La Montagna et al. ${ }^{6}$, embora tenham descrito menor densidade óssea em todas as suas pacientes, também observaram influência da idade. Diversos estudos demonstraram redução da densidade óssea na coluna, região proximal do fêmur e corpo inteiro em mulheres saudáveis, com o aumento da idade $\mathrm{i}^{16-18}$.
Ao contrário do observado em estudos anteriores, real izados em mulheres saudáveis, não observamos, em nossa casuística, qualquer redução da massa óssea, nos diversos locais examinados, com o aumento dos anos de menopausa. U ma das hi póteses aventadas para explicar nossos resultados foi que as pacientes com esclerodermia sistêmica poderiam ter, durante a menacme, níveis estrogênicos mais elevados, estando, assim, "protegidas" da perda de massa óssea. Dhillon et al. ${ }^{19}$, em 1990, ao estudarem a densidade óssea de pacientes com lúpus eritematoso sistêmico, também não verificaram qualquer redução da massa óssea na coluna dessas pacientes, mesmo em presença de corticosteróides, e levantaram a hipótese de que elas pudessem apresentar uma possível proteção hormonal. Como em nosso estudo não foi realizado o perfil hormonal, não podemos concluir que tal fato tenha ocorrido.

Serup et al. ${ }^{20}$, em 1983, sugeriram que os ovários não são acometidos pela esclerodermia sistêmica. No entanto, também esses autores não dosaram os hormônios sexuais em mulheres com essa doença.

Até o momento, poucos trabalhos foram realizados verificando a influência hormonal sobre a esclerodermia sistêmica. Existem apenas al guns relatos de casos de esclerodermia em pacientes com síndrome de Klinefelter ${ }^{21,22}$, e foi descrito que homens com esclerodermia sistêmica apresentam níveis mais elevados de estradiol circulante ${ }^{23}$.

Em nossa casuística, houve correlação negativa entre o tempo de doença e a densidade óssea apenas no colo do fêmur, não ocorrendo o mesmo nas outras regiões analisadas. Esses resultados diferem dos encontrados por La Montagna et al. ${ }^{6}$ e Serup et al. ${ }^{5}$, que não observaram correlação do tempo de duração da doença com a densidade óssea medida do antebraço. Embora alterações da força muscular não tenham sido observadas ao exame clínico das pacientes, bem como a dosagem sérica das enzimas musculares e exames el etroneuromiográficos não tenham sido real izados, a miopatia que pode ocorrer nessa doença poderia ter levado à diminuição da densidade óssea no col o femoral. Pocock et al. ${ }^{24}$, em 1989, demonstraram, em mulheres saudáveis, que a tensão muscular, o preparo físico e o peso, independente da idade, influenciam a massa óssea do colo do fêmur.

Ao se estudar a densidade óssea em um determinado grupo de indivíduos com uma doença específica, outros aspectos, além da idade, anos de menopausa e tempo de doença, devem ser, também, analisados, tais como peso, altura, índice de massa corporal, ingestão de cálcio e paridade.

O peso e altura das pacientes não foram diferentes das mulheres-controle saudáveis. Nenhuma pacien- 
te apresentou índice de massa corporal inferior a $20 \mathrm{~kg} / \mathrm{m}^{2}$. A o contrário, a média desseíndice foi el evada em nossas pacientes $\left(27 \pm 4 \mathrm{~kg} / \mathrm{m}^{2}\right)$, indicando tendência à obesidade. A influência positiva do peso sobre a massa óssea está bem estabelecida ${ }^{24-26}$. Portanto, a tendência à obesidade observada no grupo de pacientes estudadas pode ter atuado como fator de proteção, determinando maiores densidades ósseas nas regiões analisadas.

Verificou-se que 20 das 25 pacientes consumiam mais que dois copos de leite, diariamente, e grandes quantidades de verduras ricas em cálcio. Oitenta e quatro por cento apresentavam distúrbios da motilidade esofagiana, algumas com sintomas como pirose e/ou dor retroesternal. Tal fato pode ter contribuído para a maior ingestão de cálcio. Este aspecto, peculiar, observado em nossa casuística, deve ser considerado, pois pode ter atuado positivamente sobre a massa óssea das pacientes. Matkovic et al. ${ }^{27}$, em 1979, demonstraram que indivíduos normais, com maior ingestão de cálcio alimentar, possuíam densidade óssea maior do que aqueles que não tiveram a mesma ingestão. Todavia, ao estudarmos, isoladamente, o conteúdo mineral das cinco pacientes com menor ingestão de cálcio, não observamos diferença entre a média das densidades ósseas dessas pacientes, que variou entre 0,97 e $1,08 \mathrm{~g} / \mathrm{cm}^{2}$, semel hante às das outras pacientes.

A nuliparidade tem sido descrita como possível causa de menor massa óssea ${ }^{28,29}$. Diversos autores ${ }^{30-}$ 32 descreveram menor fertilidade em mulheres com esclerodermia sistêmica, e que a infertilidade pode preceder, durante vários anos, as primeiras manifestações clínicas dessa doença. Até o início do presente estudo, seis (25\%) das pacientes nunca haviam engravidado. Esses dados são semelhantes aos observados por Steen et al. ${ }^{31}$, em 1988, que relataram $35 \%$ de nuliparidade em pacientes com esclerodermia, com idade inferior a 45 anos. Em nossa casuística, as nuligestas apresentaram menor densidade óssea no fêmur proximal (colo, triângulo de Ward e trocanter), quando comparadas com as que engravidaram, de forma semelhante ao descrito em mulheres saudáveis nulíparas ${ }^{28,29}$.

E mbora as médi as de idade, anos de menopausa e tempo de evol ução da doença tenham sido maiores nas paci entes com a forma limitada, as médias das densidades ósseas, nas diferentes regiões anal isadas dessas pacientes, não foram diferentes daquelas encontradas nas pacientes com forma difusa. Esses dados sugerem que a forma de esclerodermia não exerce qualquer influência sobre a densidade óssea. Esses resultados concordam com os descritos por La Montagna et al. ${ }^{6}$ Além disso, Serup e Hadgrup ${ }^{33}$ e Matsuoka et al. ${ }^{34}$ relataram que a extensão do comprometimento cutâneo na esclerodermia não altera o metabolismo de vitamina $D$.

As pacientes com calcinose exibiram menor densidade óssea no colo do fêmur, triângulo de Ward e trocanter, quando comparadas àquelas sem calcinose. $\mathrm{Em}$ nosso estudo, observou-se que os níveis séricos de cálcio, fósforo, fosfatase alcalina e creatinina, bem como o cál ci o urinário de 24 horas, eram normais, sugerindo que o metabolismo ósseo das pacientes estudadas não estava alterado. Serup et al. ${ }^{5}$ descreveram menor densidade óssea do terço distal do rádio, naquelas com calcinose, e também não observaram alterações do metabolismo de cál cio e fósforo. Entretanto, ao analisarmos as características clínicas e demográficas das pacientes com cal cinose, constatamos que eram mais idosas e estavam na menopausa há mais tempo que aquelas sem cal cinose. Esses dados sugerem que as menores densidades ósseas encontradas na região proximal do fêmur, provavel mente, deveram-se mais ao envel hecimento propriamente dito do que à presença ou não de calcinose.

Não houve relação entre a reabsorção das falanges distais e a densidade óssea. Esse resultado contraria a hipótese proposta por Blocka et al. ${ }^{3}$, que sugeriram a existência de um processo reabsortivo ósseo generalizado na esclerodermia sistêmica.

Não houve diferença significante entre as densidades ósseas dos diversos locais estudados dos pacientes com e sem redução do índice de Nordin. Esses dados sugerem que a osteopenia em mãos, na esclerodermia sistêmica, está associada mais a fenômenos regionais, tais como tendência à imobilidade causada pela fibrose dérmica, isquemia crônica decorrente da endarterite obstrutiva da microcirculação e reabsortiva local ${ }^{35-37}$ do que a qualquer alteração sistêmica propriamente dita.

Outras hipóteses que podem ser aventadas para justificar a ausência de perda de massa óssea, na grande maioria de nossas pacientes, poderiam estar associadas à fisiopatologia intrínseca da própria esclerodermia. Tanto os osteoblastos como os fibroblastos originam-se da mesma célula mesenquimal, presente no estroma da medula óssea, e têm como principal produto o colágeno tipo l . Além disso, quando sob ação de determinadas citocinas, podem apresentar respostas semel hantes. Estudos in vitro demonstraram que a interleucina 4 (IL-4), a somatomedina C (IGF-1) e o transforming growth factor beta (TGF- $\beta$ ) desencadeiam maior síntese de colágeno por ambas as células. J á foram descritos, em pacientes com esclerodermia, níveis séricos el evados de IL-4 e níveis de IGF-1 no limite superior da normalidade ${ }^{38-40}$. 
O TGF- $\beta$ é produzido, principalmente, pelos grânulos alfa das plaquetas que, nos pacientes com esclerodermia, estão ativados e com maior tendência à agregabilidade ${ }^{41,42}$. O TGF $-\beta$ pode, também, ser gerado pelas lesões ativas da esclerodermia, decorrente da ativação dos macrófagos e linfócitos $\mathrm{T}^{42}$. $\mathrm{Na}$ esclerodermia o TGF- $\beta$ é capaz de estimular a angiogênese e aumentar a produção de colágeno, fibronectina e de matriz extracelular pelos fibroblastos ${ }^{40}$. No teci do ósseo, favorece a formação óssea, estimulando a multiplicação, maturação e atividade dos osteoblastos ${ }^{42}$. Além disso, a síntese de TGF- $\beta$ é fortemente estimulada pelo fator de crescimento derivado de plaquetas (PDGF), que se encontra elevado no soro de pacientes com esclerodermia ${ }^{43}$.

Por outro lado, certas citocinas, como a interleucina 1 (IL-1) e o fator de necrose tumoral alfa (TNF- $\alpha$ ), que aumentam a reabsorção óssea, são encontradas nos locais de maior atividade da doença $^{37,39}$, o que justificaria o encontro radiológico de osteopenia e osteólise regionais (mãos, pés, ramo da mandíbula, costelas, etc.). Entretanto, as citocinas mencionadas não teriam ação sistêmica em nível ósseo, visto que os níveis séricos estão normais em pacientes com esclerodermia sistêmi $\mathrm{ca}^{39}$.

A partir dos dados expostos, poderíamos supor que a somatória das citocinas agiria em direções opostas, em pacientes com esclerodermia. Na pele, atuariam negativamente, provocando o aparecimento das lesões e dos sintomas característicos da esclerodermia, e, no tecido ósseo, exerceriam ação protetora, preservando a massa óssea. I mportante ressaltar que essas considerações foram aventadas apenas para justificar a ausência de perda de massa óssea encontrada em nossas pacientes, e que nenhuma das referidas citocinas foram medidas no presente estudo.

No sentido de se esclarecer as relações entre os fatores envolvidos na fisiopatologia da esclerodermia e suas possíveis ações sobre o tecido ósseo, poderiam, ainda, ser realizados novos estudos, com maior número de pacientes, distribuição mais uniforme das diferentes formas clínicas de esclerodermia, avaliação do perfil hormonal, dosagem de outros marcadores bioquímicos do metabolismo ósseo e das citocinas mencionadas.

\section{SUMMARY}

\section{Bone density in systemic sclerosis (sclero- derma) patients}

Background - Osteopenia in systemic sclerosis (scleroderma) patients was reported in X-ray studies of hands and by proximal and distal forearm bone mass measurement. It has been suggested that bone loss in these patients might be due to chronic ischemia, immobilization and early menopause. Nevertheless it is not established if these patients present generalized osteopenia. To shed light into this point we studied bone mineral density in the spine, proximal femur and total body in patients with systemic sclerosis.

Patients and Method - Twenty-five Caucasian women were evaluated. Mean age of patients was $48 \pm 12$ years and mean time of disease was $7 \pm 7$ years; 13 were postmenopausal ( $8 \pm 8$ years). Bone mass was measured in the spine, proximal femur and total body by using a dual-photon absorptiometry with $X$ rays source (Lunar - Model DPX).

Results - Bone mass in different sites was not statistically different from the age-matched control healthy women. Mean bone mass of patients with limited form was not different from patients with diffuse form of systemic sclerosis. Patients with calcinosis had lower bone mass at proximal femur than those without this al teration.

Concl usions - Patients with systemic sclerosis do not present bone loss and this di sease is not a risk factor for generalized osteoporosis. [Rev Ass Med Brasil 1997; 43(1): 40-6.]

KEY WORDS: Bone mineral density. Osteoporosis. Systemic sclerosis.

\section{REFERÊNCIAS BIBLIOGRÁFICAS}

1. Basset LW, Blocka KLN, Furst DE et al. Skeletal findings in progressive systemic sclerosis (scleroderma). AJ R 1981; 136: 1.121-6.

2. Doyle T, Littlejohn G, Miller M, Barnett A. The radiographic changes of scleroderma in the hands. Aust Radiol 1990; 347: 53-8.

3. Blocka KLN, Basset LW, Furst DE et al. The artropathy of advanced progressive systemic sclerosis - a radiographic survey. Arthritis Rheum 1981; 24 : 874-84.

4. Khonstanteen I, Wright B, Russell ML. Localized bone resorption in systemic sclerosis. J Rheumatol 1988; 15: 1.435-37.

5. Serup J , Hagdrup H, Tvedegaard E. Bone mineral content in systemic sclerosis criteria for the classification of systemic sclerosis (scleroderma). Arthritis Rheum 1980; 23: 581-90.

6. La Montagna G, Vatti M, Valentini G, Tirri G. Osteopenia in systemic sclerosis: evidence of a participating role of earlier menopause. Clin Rheumatol 1991; 10: 18-22.

7. Rodnam GP. Progressive systemic sclerosis (scleroderma). In Hollander J L, McCarty J r DJ (eds): Arthritis and allied conditions. Philadel phia, Lea \& Febiger 1979; 962-8.

8. Subcomittee for scleroderma criteria of the American Rheumatism Association. Preliminary criteria for the classification of systemic sclerosis (scleroderma). Arthritis Rheum 1980; 23 : 581-90.

9. Nordin BEC. Osteoporosis with particular reference to the menopause. In N ordin BEC, Riggs BL, Teiltel baum SL (eds): The osteoporotic syndrome. Orlando, Grune \& Stration, 1983; $13-43$. 
10. Szejnfeld VL, Aldrighi J M, Ferraz MB, Atra E. Spine and femur density using dual photon absorptiometry in Brazilian white women. J Bone Min Res 1991; 5(15): abstract 191.

11. Giordano M, Valentini G, Migliaresi S et al. Different antibody and different prognosis in patients with Scleroderma with various extents of skin sclerosis. J Rheumatol 1986; 13: 911-6.

12. Schlenker RA, Von Sieggen WW. The distribution of cortical and trabecular bone mass along the lenghts of the radius and ulna and implications for in vivo bone mass measurements. Calcif Tissue Res 1970; 20: 41-3.

13. Resnick D, Sartoris $D$ J . Dual energy radiographic absorptiometry for bone densitometry. Current status end perspectives. AJ R 1989; 152: 241-6.

14. Wahner HW, Eastell R, Riggs BL. Bone mineral density of the radius where do we stand (editorial)? J Nucl Med 1985; 26:1.339-41.

15. Mazess RB, Barden HS, Ettinger M. Radial and spinal bone mineral density in patient population. Arthritis Rheum 1988; 31: 891-7.

16. Aloia J F, Vaswani A, Ross P, Cohn SH. Aging bone loss from femur, spine, radius and total skel eton. Metabolism 1990; 39: 1.144-50.

17. Mazess RB. On aging bone loss. Clin Orthop 1982; 165: 239-45

18. Riggs BL, Melton III LJ . Involutional osteoporosis. N Engl J Med 1986; 314: 1.676-84.

19. Dhillon VB, Davies MC, Hall MI et al. Assessment of the effect of oral corticosteroids on bone mineral density in SLE. Ann Rheum Dis 1990; 49: 624-9.

20. Serup J, Hagdrup HK. Age of menopause of females with systemic sclerosis. Acta Der Venereol (Stockh) 1983; 63: 71-3.

21. De Keiser $P$, Mielants $H$, Veys $E M$. Klinefelter syndrome and scleroderma. J Rheumatol 1989; 16: 1.613-4.

22. Kobayashi S, Shimamoto T, Taniguchi O et al. Is Klinefelter syndrome associated with progressive systemic sclerosis? Report of a case and review of the literature. Clin Rheumatol 1991; 10: 84-6.

23. J emec GBE, Sindrup JH. Circulating androgens in male patients suffering from systemic sclerosis. Arch Dermatol Res 1991; 283: 289-91.

24. Pocock N, Eisman J , Gwinn T et al. Muscle strenght, physical fitness and weight but not age predict femoral neck bone mass. J Bone Min Res 1989; 4: 441-7.

25. Frunar A, Moldsin ID, Geola $F$ et al. Relationship of fasting urinary calcium in circulating estrogens and body weight in postmenopausal women. J Clin Endocrinol 1980; 50: 70-5.

26. Heliovara M, Aromar A. Parity and obesity. J Epidemiol Commun Health 1981; 35: 197-9.
27. Matkovic V, Kostial K, Simonovic L. Bone status and fracture rates in two regions of Yugoslavia. Am J Clin Nutr 1979; 32: 540-9.

28. Lindsay R, Herrington BS, Tohme J . Reproductive history and bone mass in women. J Bone Min Res 1986; 1 (1): 248.

29. Lindsay R. Sex steroids in pathogenesis and prevention of osteoporosis. In Riggs BL, Melton III J L (eds): Osteoporosis: prevention, diagnosis and treatment. New York, Raven Press 1988; 333-58.

30. Silman AJ, Black C. Increased incidence of spontaneous abortion and infertility in women with scleroderma before onset: a controlled study. Ann Rheum Dis 1988; 47: 441-4.

31. Steen VD, Conte C, Day N et al. Pregnancy in women with systemic sclerosis. Arthritis Rheum 1989; 32: 151-7.

32. Stenever MA, Nug ABP. Scleroderma of uterine cervix. Am J Obstet Gynecol 1970; 107: 965-6.

33. Serup J, Hagdrup H. Vitamin D metabolites in generalized scleroderma: evidence of normal cutaneous and intestinal supply with vitamin D. Acta Der Venerol (Stockh) 1985; 65: 343-5.

34. Matsuoka LY, Dannemberg MJ , Wortsman J et al. Cutaneous vitamin D3 formation in progressive systemic sclerosis. J Rheumatol 1991; 18: 1.196-8.

35. Claman HN. On scleroderma, mast cells, endothelial cells and fibroblasts. J AMA 1989; 262: 1.206-9.

36. McKenna MJ , Frame B. The mast cell and bone. Clin Orthop Rel Res 1985; 200: 226-33.

37. Umehara $\mathrm{H}$, Kumagai $\mathrm{S}$, Murakami $\mathrm{M}$ et al. Enhanced production of interleukin-1 and tumo necrosis factor alfa by cultured peripheral blood monocytes from patients with scleroderma. Arthritis Rheum 1990; 33: 893-7.

38. Famularo G, Procopio A, Giacomelli R et al. Soluble interleukin-2 receptor, interleukin-2, interleukin-4 in sera and supernatants from patients with progressive systemic sclerosis. Clin Exp Immunol 1990; 81: 368-72.

39. Needleman BW, Wigley FM, Stair RW. Interleukin-1, interleukin-2, interleukin-6, tumor necrosis alfa and interferon gama level in sera from patients with scleroderma. Arthritis Rheum 1992; 35: 67-72.

40. Falanga V, Altman RD., Roth MJ . Plasma somatomedin-C levels in systemic sclerosis. Br J Dermatol 1988; 119: 639-42.

41. Kahaleh MB, Osborn L, LeRoy EC. Elevated levels of circulating platelets agregates and beta-tromboglobulin in scleroderma. Ann Intern Med 1982; 96: 610-3.

42. Smith EA, LeRoy EC. A possible role for transforming growth factor-beta in systemic sclerosis. J Invest Dermatol 1990; 95: 125-7.

43. Pandolfi A, Florita M, Altomare G et al. Increased plasma levels of platelet derived growth factor activity in patients with progressive systemic sclerosis. PSEBM 1989; 191: 1-4. 